\title{
Identification of material parameters using indentation test -study of the in- trinsic dimensionality of P-h curves and residual imprints
}

\author{
Liang Meng ${ }^{1, a}$, Piotr Breitkopf ${ }^{1}$ and Guenhael Le Quilliec $^{2}$ \\ ${ }^{1}$ Sorbonne Universités, Université de Technologie de Compiègne \\ UMR UTC-CNRS 7337, Compiègne, France \\ ${ }^{2}$ University of Tours, E.A. 2640 Laboratoire de Mécanique et Rhéologie, Tour Cedex, France
}

\begin{abstract}
Instrumented indentation test has been used to determine material parameters with two different sources of information. Based on the recent shape-manifold identification approach, we formally demonstrate, in the scope of manifold, the non-uniqueness of the solution to the inverse problem based on load-displacement curves. The identifiability is also compared for P-h curve and imprint mapping, highlighting the manifold's ability to estimate the maximum number of independent material parameters that may be determined with a given experimental setup.
\end{abstract}

\section{Introduction}

Instrumented indentation is a popular technique used for the assessment of material properties. Fruitful research results have been reported [1-5] for a variety of materials, such as metals, alloys, ceramic, concrete and bone, among others. In a typical identification procedure, it is expected that there exists a one-to-one correspondence between the input pattern (usually the indentation curve, also called a P-h curve) and the desired material parameters defining a postulated constitutive law. Unfortunately, the indentation problem is frequently ill-posed, and the existence of different "sibling" materials with distinct material properties but almost identical indentation behaviors has been reported more than once [6-8].

The often used inverse method, employing Finite Element (FE) simulation and optimization algorithms, tries to estimate the material parameters by minimizing the discrepancy between measured and simulated quantities. However, the uniqueness of the solution still remains a matter of discussion since different material parameter sets may give almost identical indentation curves [7, 8]. Therefore, it is of high interest to improve the reliability of the inverse analysis by involving more information for identification. Accompanied by the development of highresolution Scanning probe microscopy (e.g., Atomic Force Microscopy (AFM)), [2, 9, 10] reported several methods for assessing material properties, taking into consideration not only the indentation curve, but also the residual imprint measured with high precision and accuracy, such that material's unknown properties are evaluated in a much more elegant manner in that more parameters are identifiable. Other solutions, like adopting indenters with complex profiles (i.e., a dual sharp indenter), were also proposed to

\footnotetext{
${ }^{\mathrm{a} C}$ Corresponding author: liang.meng@utc.fr
}

alleviate the non-uniqueness property of the identification problem[11-13].

More recently, due to the significant development of manifold learning algorithms and its applications in the field of computational and applied mechanics, biomechanics, and especially in material science[14-18], our most recent work [19] recommended that the identification procedure be carried out within a $\alpha$-space constructed by using only the residual imprint of an indentation test. The core idea is based on the fundamental hypothesis that, there exists a low-dimensional space in which the original higherdimensional data could be embedded and the identification is implemented over a manifold defined within the constructed shape space. The efficacity of this approach has been demonstrated by successfully evaluating two hardening properties of a piece of C100 steel and an AU4G alloy.

Regarding the remarkable advancements in material characterization, to the knowledge of the authors, the number of parameters that can be probed by an instrumented indentation test has never been systematically investigated, despite the awareness of the presence of uniqueness. In this work, we extend the aforementioned imprint-based manifold identification approach to the measured indentation curve to investigate the non-uniqueness phenomenon. In addition, the manifold is also studied in order to obtain a cursory estimation of the number of parameters that can be identified.

\section{Material characterization using manifold learning}

In our former work [19], we proposed an original approach for evaluating diverse material properties solely based on the residual imprint after withdrawing the indenter. This information, usually high-dimensional, was not employed 
in calculating the point-wise distance so as to characterize the discrepancy between simulated and experimental measured quantities. Instead, we first seek a low-dimensional embedded domain in which it can be described in a more compact pattern. There is a common sense notion in the community of signal processing that "Real life data does not uniformly fill the space in which it is represented". Instead, their distributions are observed to concentrate to a manifold (linear/non-linear) of lower intrinsic dimensionality.

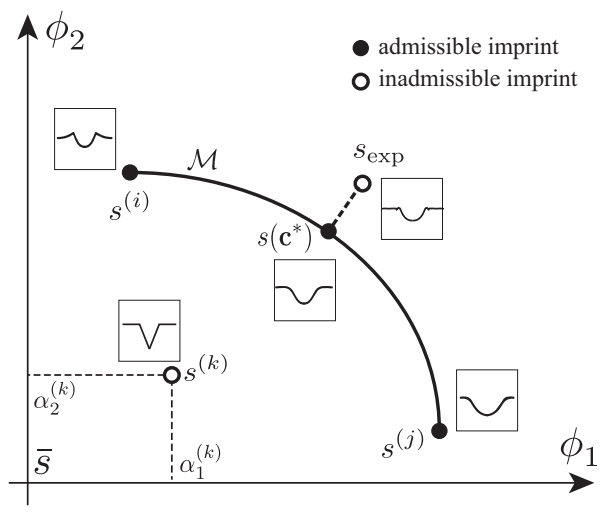

Figure 1. Illustration of admissible/inadmissible imprints in a low dimensional embedded space.

The manifold is generally problem-dependent. For an indentation test, which is our case, the manifold connects all of these simulated imprints together. As a consequence, we are able to distinguish between admissible/inadmissible shapes by referring them to the manifold. Generally, we consider an imprint as admissible under the condition that it is attainable by manipulating the parameter set $\mathbf{c}$ in a postulated constitutive law. Two admissible imprints $\mathbf{s}^{(i)}$ and $\mathbf{s}^{(j)}$, as noted by solid circles in Fig.1, lie exactly on the the manifold $\mathcal{M}$. On the other hand, all of those imprints outside the manifold may then be inadmissible, e.g., $\mathbf{s}^{(k)}$, marked by a round circle. It is worth noting that the experimental imprint $\mathbf{s}_{\mathrm{e} x p}$ is inadmissible in consideration of measurement noise and model error.

In practice, find the proper embedded system can be done in various ways $[18,20,21]$, and we adopted the Principal Component Analysis (PCA), which is routinely used in image analysis [22]. Note here, the embedded space is a POD coefficient space, the origin of which is the mean imprint $\overline{\mathbf{s}}$ of all these in the database, and its principle axis are orthogonal basis derived from PCA. Thus the embedded system is also called an $\alpha$-space and the hyper-surface an $\alpha$-manifold. Their constructions were detailed in [19] thus omitted here.

The identification procedure is quite straightforward in the constructed $\alpha$-space: find the parameter set that gives an admissible shape $\mathbf{s}\left(\mathbf{c}^{*}\right)$ on the manifold $\mathcal{M}$ which is closest (minimum distance) to the experimental shape

$$
\mathbf{c}^{*}=\operatorname{Argmin}\left\|\left(\alpha_{\exp }-\alpha(\mathbf{c})\right)\right\|, \quad \alpha(\mathbf{c}) \in \mathcal{M},
$$

here $\alpha_{\exp }$ is the coordinate of the experimental imprint in this shape space; the constraint $\alpha(\mathbf{c}) \in \mathcal{M}$ confines automatically that the imprint shape should be an admissible one. We summarize the complete manifold-based identification processus as follows:

1. Create a database of imprints by a proper Design Of Experiment (DOE) varying material parameters in a constitutive law;

2. Find the embedded lower-dimensional space by PCA and project the imprint database into the new system;

3 . Interpolate a manifold in $\alpha$-space and built a one-toone relationship between the design space and the point on manifold;

4. Project experimental imprint on the manifold and identify material parameters referring to Eq.(1).

It is worth mentioning that the pair-wise distance between two different imprint shapes is transformed into a single Euclidean distance in $\alpha$-space.

\section{Description of measured quantities by indentation}

(a)
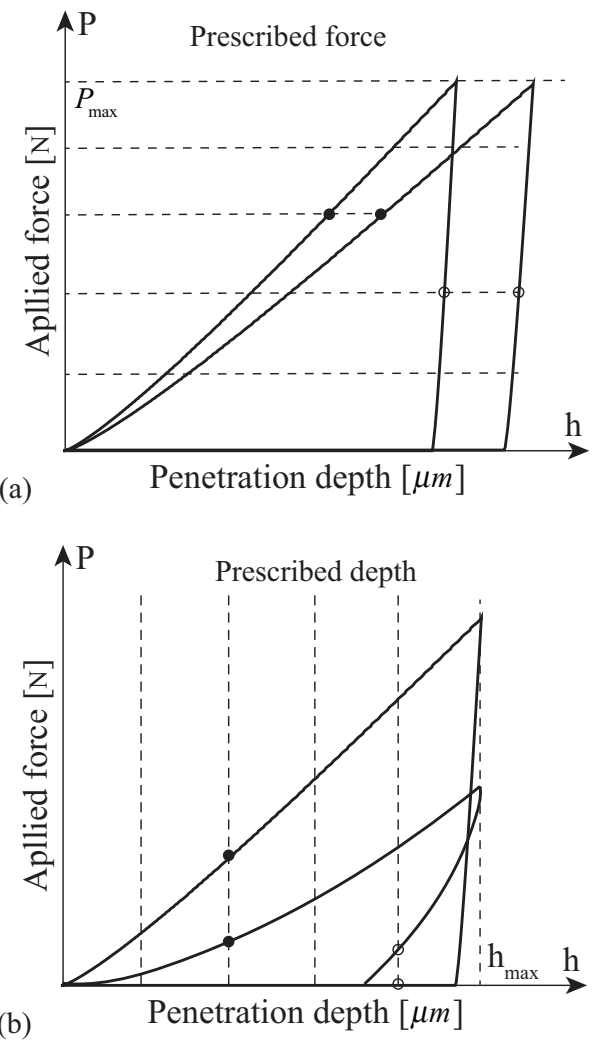

Figure 2. Characterization of different indentation curves.

The manifold-based identification approach does not restrict itself to imprint mapping only. We can also consider the obtained P-h curve as a special 'imprint' since the ultimate goal is to distinguish these curves/shapes one from another.

Unlike imprint mapping, $\mathrm{P}-\mathrm{h}$ curve is a multivalued function and it makes no sense to compare the loading phase of one curve with the unloading phase of another in identification procedure, thus these two phases should be properly separated. Take a typical indentation curve obtained by a prescribed force as an example, the two parts are characterized by the penetration depths at a series of 

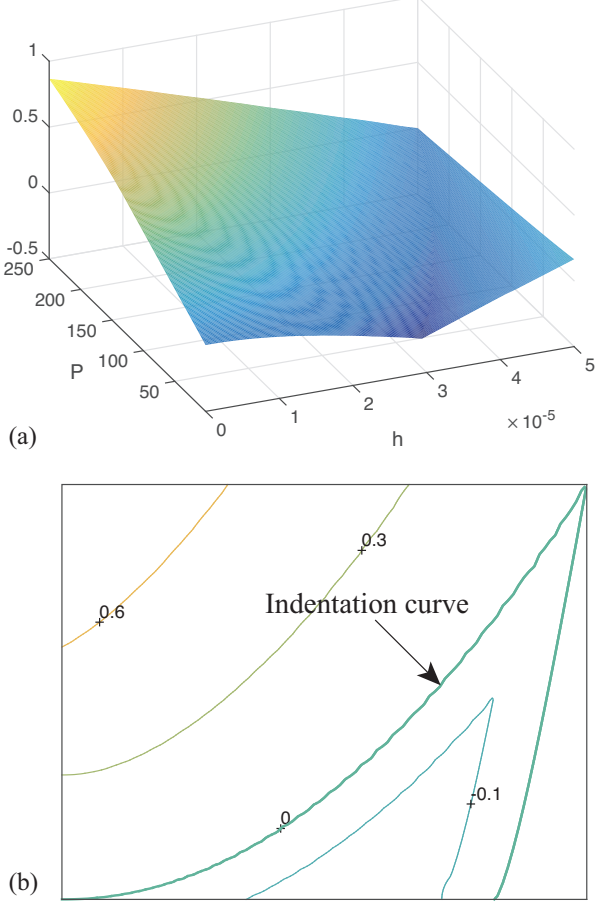

Figure 3. A typical indentation curve described by the zero isocontour of a Level-set function.

given forces, noted by $\mathbf{s}_{l}$ and $\mathbf{s}_{u}$, respectively. Then the whole curve is described by a vector which concatenates the two, giving $\mathbf{s}_{c}=\left[\mathbf{s}_{l}, \mathbf{s}_{u}\right]$, guaranteeing the comparability of different curves. For the case where a maximum penetration depth is predefined, the same routine should be followed except that we compare forces for different penetration depths. For the latter, it should be noticed also that the unloading part includes also the segment that overlies on the $h$-axis. Fig. 2 illustrates the characterization of the P-h curves obtained from two experimental set ups: (a) prescribed force; (b) prescribed penetration depth. By consequence, our manifold protocol can be extended to indentation curve also, allowing for a comparison between the identifiabilities of two source of information.

This concatenating description, while feasible, is setupdependent. Thus it is of great interest to describe the P-h curve with an Eulerian representation, examples of which may include pixel/voxel maps, and the more popular Levelset approach which is first proposed in [23]. The basis idea is the use of a background grid points and define the Level-set function simply as the signed minimum distance of these points to the curve. By convention, the distance is positive if the point lies outside the curve while negative for inside ones. As a result, the zero iso-contour describes the boundary of the curve. A typical indentation curve which is characterized by such method can be found in Fig.3.

\section{Numerical examples}

\subsection{Forward analysis using FE}

First, by adopting the same FE model used in our last work [19], two databases, each containing $21 \times 21=441$ samples, were built up for indentation curves and imprint mappings by simulations with various hardening parameter combinations. The simulation parameters are equalspaced sampled from the design domain ( $n \in[0.2,0.3]$, $\left.\sigma_{y} \in[200,240]\right)$ allowing a straightforward demonstration of connectivity in embedded $\alpha$-space.

The material hardening law is characterized by two parameters: elastic limit $\sigma_{y}$ and hardening coefficient $n$, having the form

$$
\sigma=\sigma_{y}\left(\frac{E}{\sigma_{y}}\right)^{n} \varepsilon^{n} .
$$

\subsection{Identifiability analysis using manifold}

In this section, the two database were employed separately so as to detect the identifiability of P-h curve and imprint mapping. It should be noticed that, the bellowing results are obtained from purely numerical data, and the experimental imprint is chosen from one of the 441 simulation results and no noise is introduced.

Errors of identification criterions employing different measured quantities are first included in Fig.4. Clearly, for conventional point-wise distance error, the parameter sets $\left(\sigma_{y}, n\right)$ having almost the same minimal error are found in a thin-long valley. This is highly consistent with the one observed by [24]. Similar result is also obtained for the imprint mapping.

On the other hand, by adopting the innovate identification method referring to the manifold, the Euclidean distance between the measured quantities and the corresponding experimental one is calculated. Its distribution is found in the second row of Fig.4. To our delight, from the isoline of the error, we probably yield a convergent iterative value. However, in this situation, there is no way to guarantee that the solution is a global minimal rather than a local one. Similar result is obtained if we employ the same manifold approach for P-h curves. Thus we would like to conclude that, the manifold based method takes more advantage of the origin measured data, while no clear difference is observed for P-h curve and imprint mapping.

Moreover, since the manifold method interprets well the measured quantities compared with conventional method, it is of great interest to compare the two manifolds constructed from P-h curve and imprint mapping respectively. It is observed from Fig.5.(a) and Fig.6.(a) that both the manifolds are thin and long, illustrating that only one of the sensitivities with respect to the two parameters has a significant value and the other is almost insensible. This explain quite clear the reason why it is difficulty to identify the two parameters simultaneously.

That said, slight difference are also observed for the two manifolds. We noticed that the $\alpha$-manifold of the imprint shapes seems to expand along two directions despite its long-thin property. Motivated by this, we consider to normalize the $\alpha$-coordinates of each embedded point in shape space to bring the error sensitivity with respect to the two parameters to a comparable level. The scaled $\alpha$-manifolds are given in Fig.5.(b) and Fig.6.(b).

Clearly, the scaled manifold proved that the imprint interprets the material properties in the most elegant man- 

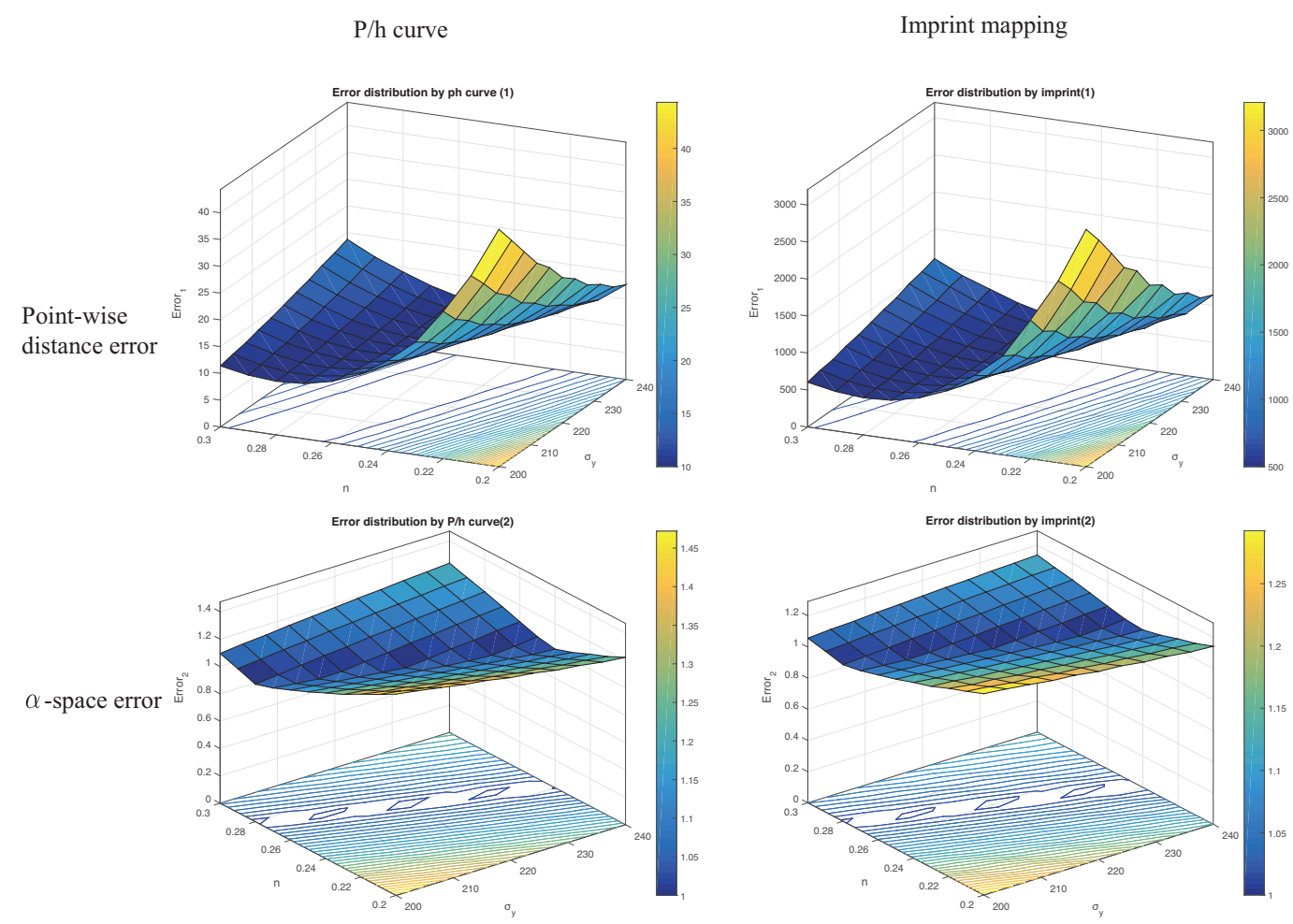

Figure 4. Comparisons of error distribution within different spaces employing different information sources.

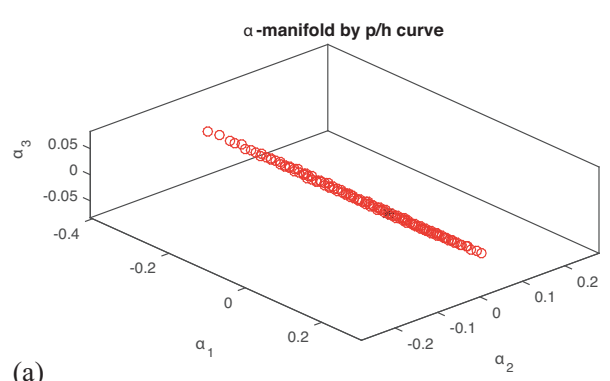

(a)

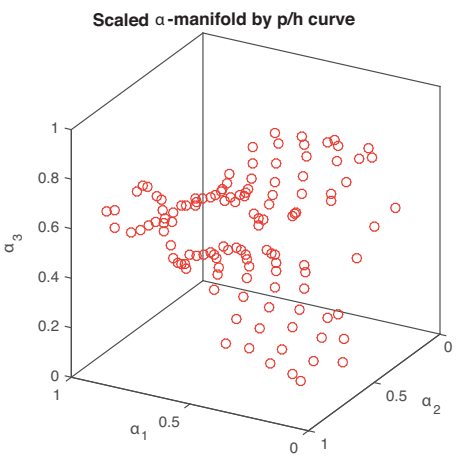

Figure 5. (a)Original manifold profile; (b) Manifold in normalized $\alpha$-coordinate system obtained from indentation P-h curve.

ner since the manifold has a dimensionality of 2 , which is right the number of parameters for the construction of database. Also, similar with their distributions in parametric space, different imprints are embedded regularly in the shape space (Fig.7 left), allowing the identification of the parameter set. Contrarily, the embedding of P-h curve is kind of disordered make it inappropriate for material charcterization.

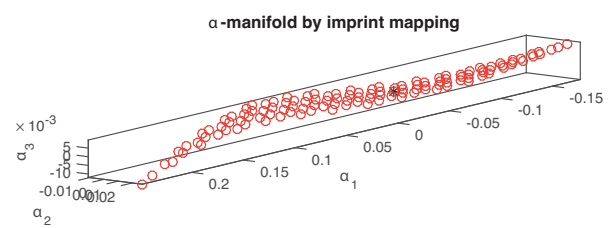

(a)

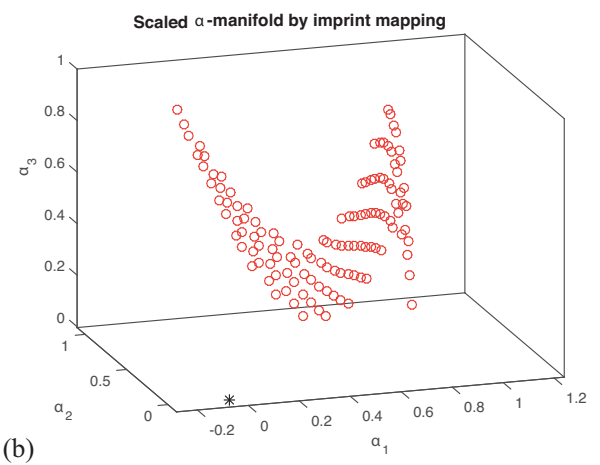

Figure 6. (a)Original manifold profile; (b) Manifold in normalized $\alpha$-coordinate system obtained from imprint mapping.

\section{Conclusion}

In this work, we demonstrated the identifiability of different measured indentation quantities using a shape manifold and observed that the residual imprint of an indentation test outperforms the P-h curve during identification. In addition, the identification procedure based on the manifold has the additional ability to estimate the maximum number of identifiable parameters $d_{i n}$, especially for those with a dimensionality $d_{\text {in }}<=3$. This outstanding property of the 

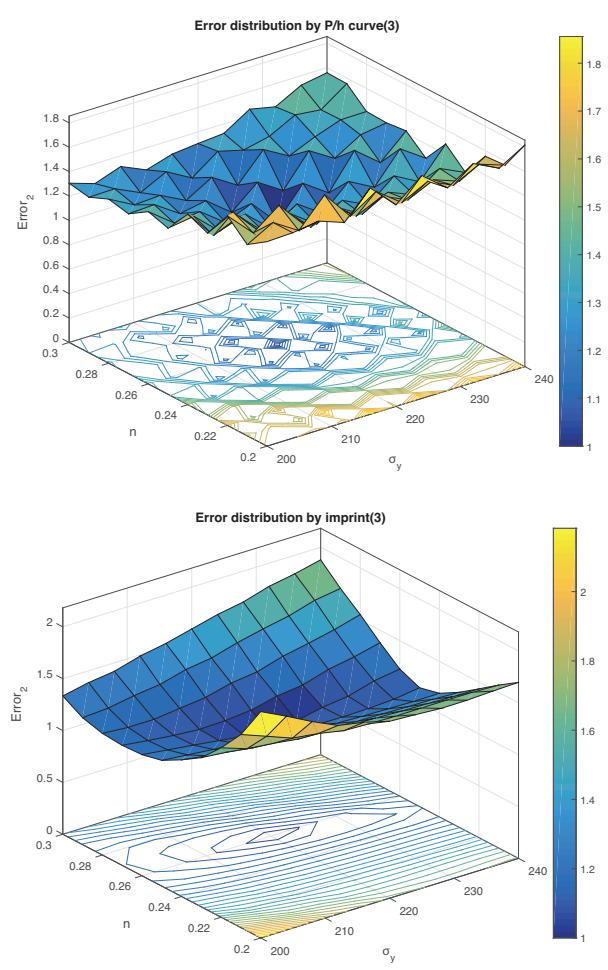

Figure 7. Error distributions computed from scaled $\alpha$-manifold.

manifold could provide valuable guidance for designing the indentation test and choosing the quantities to be measured.

That said, for complex material law which involves more parameters, the manifold can no longer be visualized. Thus the quantitive estimation of intrinsic dimensionality is of great necessity.

\section{References}

1. C.K. Moy, M. Bocciarelli, S.P. Ringer, G. Ranzi, Materials Science and Engineering: A 529, 119 (2011)

2. M. Bocciarelli, G. Bolzon, Materials Science and Engineering: A 448, 303 (2007)

3. S. Kucharski, Z. Mroz, Journal of engineering materials and technology 123, 245 (2001)

4. D. Carnelli, R. Lucchini, M. Ponzoni, R. Contro, P. Vena, Journal of biomechanics 44, 1852 (2011)

5. M. Rodríguez, J.M. Molina-Aldareguía, C. González, J. LLorca, Acta Materialia 60, 3953 (2012)
6. Y.T. Cheng, C.M. Cheng, Journal of Materials Research 14, 3493 (1999)

7. K. Tho, S. Swaddiwudhipong, Z. Liu, K. Zeng, J. Hua, Journal of materials research 19, 2498 (2004)

8. J. Alkorta, J. Martinez-Esnaola, J.G. Sevillano, Journal of materials research 20, 432 (2005)

9. G. Bolzon, G. Maier, M. Panico, International Journal of Solids and Structures 41, 2957 (2004)

10. M. Bocciarelli, G. Bolzon, G. Maier, Mechanics of Materials 37, 855 (2005)

11. M. Futakawa, T. Wakui, Y. Tanabe, I. Ioka, Journal of Materials Research 16, 2283 (2001)

12. J.L. Bucaille, S. Stauss, E. Felder, J. Michler, Acta materialia 51, 1663 (2003)

13. Y. Cao, N. Huber, Journal of materials research 21, 1810 (2006)

14. B. Raghavan, P. Breitkopf, Y. Tourbier, P. Villon, Structural \& Multidisciplinary Optimization 48, 987 (2013)

15. B. Raghavan, L. Xia, P. Breitkopf, A. Rassineux, P. Villon, Computer Methods in Applied Mechanics and Engineering 265, 174 (2013)

16. B. Raghavan, G. Le Quilliec, P. Breitkopf, A. Rassineux, J.M. Roelandt, P. Villon, International Journal of Material Forming 7, 487 (2014)

17. G. Le Quilliec, B. Raghavan, P. Breitkopf, Computer Methods in Applied Mechanics and Engineering 285, 621 (2015)

18. D. González, E. Cueto, F. Chinesta, Annals of biomedical engineering pp. 1-11 (2015)

19. L. Meng, P. Breitkopf, B. Raghavan, G. Mauvoisin, O. Bartier, X. Hernot, Computer Methods in Applied Mechanics and Engineering (2015)

20. L.K. Saul, S.T. Roweis, unpublished. Available at: http://www. cs. toronto. edu/ roweis/lle/publications. html (2000)

21. L.K. Saul, K.Q. Weinberger, J.H. Ham, F. Sha, D.D. Lee, Semisupervised learning pp. 293-308 (2006)

22. I. Jolliffe, Principal component analysis (Wiley Online Library, 2002)

23. S. Osher, J.A. Sethian, Journal of computational physics 79, 12 (1988)

24. C. Moussa, X. Hernot, O. Bartier, G. Delattre, G. Mauvoisin, Materials Science and Engineering: A 606, 409 (2014) 